\title{
Study of the process of seed movement in the seed duct and the impact on the operation of a multi-level seeding system
}

\author{
Maksim Litvinov* \\ Federal Scientific Agroengineering VIM, 1st Institutsky proezd, 5, Moscow, 109428, Russian \\ Federation
}

\begin{abstract}
In this paper, we consider the study of the process of seed movement in the seed duct and the impact on the operation of a multi-level seeding system. Mathematical models of the movement of the seed material from the seeder to the plowshare group along the seed line are obtained. To a greater extent, the movement time is affected by the weight of the sown portion and the initial speed of the seeds when falling from the seeder. Also, in straight sections, the time depends on the geometric parameters of the seed line. In the future, the formula of the complete transfer of seeds from the seeder to the plowshare group will allow you to adjust the mode of adjusting the response time of the dosing system on a self-propelled seeder with a multi-level seeding system.
\end{abstract}

\section{Introduction}

The process of agricultural crops sowing in selection and seed production has a high degree of dependence of yield on the quality of sowing, and this dependence is determined not only by the precise execution of technologically necessary work, but also by the use of the most advanced seeders at the moment [1].

Account of kinematics of seed movement allows us to adjust the response time of dosing systems to ensure that the uniform length of dividing strips between the plots is observed.

In the selection at the II and III stages, self-propelled cassette seeders with cone seeding machines with the location of the seeding systems in the inter-axle space of the seeder are used [2]. With this arrangement, the seed lines have an increased length, since it is structurally problematic to place the seeder near the plowshares due to the small distance between the rows and the design features of the cone seeder.

The aim of the study is to determine the equation of the time of seed movement from the seeder to the soil surface to adjust the response time of the seed feeding mechanism from the hopper and the solenoid dispenser of the seeder.

\footnotetext{
*Corresponding author: mlitv@mail.ru
} 


\section{Materials and methods}

\subsection{Self-propelled selection seeder with multi-level seeding system}

A model sample of a self-propelled seeder with a multi-level seeding system with a cone seeding machine, presented in Fig. 1, was shown as a prototype for the development of a mathematical model of the process of seed movement in the seed line.

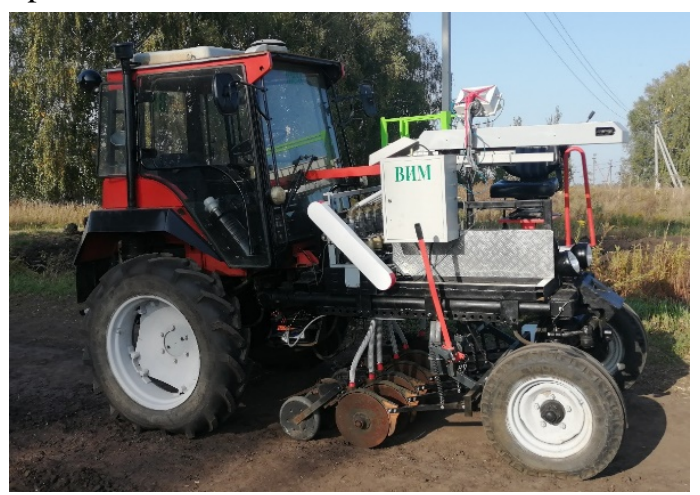

Fig. 1. Self-propelled seeder with multi-level seeding system

This model sample has several levels of control of the seeding process: adjusting the speed of the seeder taking into account jerks and wheel slippage, measuring the distance traveled, adjusting the response time of the dosing system [3].

\subsection{Kinematics of the seed movement in the seeder}

The technological process of seed material sowing can be divided into several stages (Fig.2.): 1- fall of seeds on the surface of the sowing cone, 2- fall of seeds into the seed duct, 3transport of seeds through the seed duct to the plowshare group [4].



Fig. 2. Consideration of the trajectory of the seed movement, taking into account the location of the seed line

When considering the first stage of seed movement, the driving force will be the friction force [5] of seeds on the surface of the cone ( internal friction force of seeds located above the sown seed is neglected at this stage) (Fig.3.). 


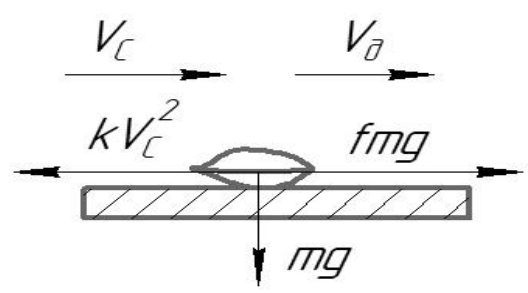

Fig. 3. Diagram of the seed movement on the surface of the distribution table

Accordingly, in this case the differential equation of motion will take the form (1).

$$
m \frac{d V_{C}}{d t}=f \cdot m \cdot g
$$

where $\mathrm{V}_{\mathrm{C}}$ - speed of absolute seed movement. Therefore, regardless of whether there is an acceleration of the surface of the sowing cone, the limiting acceleration of seeds will be determined by the expression (2)

$$
a=\frac{d V_{C}}{d t}=f \cdot g .
$$

After integrating the obtained expression under the initial conditions of movement (initial speed and the distance traveled are equal to zero $\mathrm{V}_{0}=0, \mathrm{~S}=0$ ), we can write the value of the speed of seeds and the distance traveled in the form ( 3 and 4$)$.

$$
\begin{aligned}
& V_{C}=V_{0}+f \cdot g \cdot t ; \\
& S_{C}=V_{0} \cdot t+\frac{f \cdot g \cdot t^{2}}{2} .
\end{aligned}
$$

Taking into account that the speed of the cone's movement is greater than the initial speed of the seed movement, and the friction force is less than the inertia force of the particle [6], with regard to the internal friction of seeds, to exceed the moment of giving the particle the speed of the cone's movement, the seeds move along a uniformly accelerated motion. Let us determine the time $t$ of the beginning of the seed movement at $\mathrm{V}_{\mathrm{C}}=\mathrm{V}_{\mathrm{D}}$. Using the formula (4), we substitute the variable $V_{D}$ in it and express the desired time (5).

$$
t=\frac{V_{\text {д }}-V_{0}}{f \cdot g} .
$$

Taking into account equation (4), we can write the expression of the length of the path traveled by the seeds until the moment of equalization of the seeds' speed and the cone (6).

$$
S_{C}=\frac{V_{\mathrm{A}}^{2}-V_{0}^{2}}{2 f g} .
$$

At the initial moment of time, when the speed of the seed movement is zero, the equation will take the form (7).

$$
S_{\text {C.START. }}=\frac{V_{\mathrm{A}}^{2}}{2 f g}
$$

The beginning of the relative movement of seeds, accompanied by the movement of the cone element, is described by equation (8).

$$
S_{\text {ДSTART. }}=V_{\text {Д }} \cdot t_{\text {start. }}=\frac{V_{\text {Д }}^{2}-V_{\text {д }} \cdot V_{0}}{f g}
$$


To determine the value of the absolute speed of seeds when they hit the cone, we substitute in equation (5) the value of the path traveled by the seeds until the moment of the cone's descent, determining the time value.

It is assumed that the seeds have passed the path $\mathrm{S}_{\mathrm{dis}}$, then the time of falling is determined by the formula (9).

$$
t_{\text {fall. }}=-\frac{V_{0}}{f \cdot g}+\sqrt{\frac{V_{0}^{2}}{(f \cdot g)^{2}}-\frac{2 S_{\text {fall }}}{f \cdot g}}
$$

where the rate of seed loss is determined by the expression (10).

$$
V_{\text {fall. }}=\sqrt{V_{0}^{2}+2 f \cdot g \cdot S_{\text {fall. }}}
$$

\section{Kinematics of the seed movement in the seed duct}

As can be seen from the resulting equation of the speed of fallen seeds, the speed of the cone and the path traveled by the seeds have a special effect on the increase in the speed of seeds at the time of falling. In the process under consideration, the factors of external resistance of air flow are not taken into account. To consider the third stage - the movement of the particle along the seed duct, take the seed movement in the horizontal section of the seed duct (Fig. 4.).



Fig. 4. Moving seeds in a horizontal section of the seed line

At the initial moment, the speed of seed movement was determined by the equation (10). The differential equation of seed movement is written as follows (11)

$$
m \frac{d V}{d t}=V_{C}-f \cdot m \cdot g .
$$

From the equation of motion, you can express the time of the seed movement

$$
d t=\frac{m d V}{V_{C}-f \cdot m \cdot g}=\frac{d V}{\frac{V_{C}}{m}-f \cdot g},
$$

And from the latter, integrating, we can get the formula (23)

$$
t=-m \cdot \ln \left(\frac{V_{C}}{m}-f \cdot g\right)+C,
$$

where $\mathrm{C}$ - arbitrary constant.

At an initial time the value of zero and the certain speed value $\mathrm{vc}_{\mathrm{c}}$ can express the arbitrary constant by the formula (13), then to transform the equation (13) as the equation (14).

$$
t=-m \ln \left(\frac{\frac{V_{C 1}}{m}-f \cdot g}{\frac{V_{C}}{m}-f \cdot g}\right) .
$$

Determining the speed at a given time using the formula (15) 


$$
V_{C}=-m \cdot f \cdot g-m\left(\frac{V_{C 1}}{m}-f \cdot g\right) e^{-\frac{t}{m}} .
$$

However, it is known that the speed is the time derivative of the path, therefore, to determine the path traveled, we integrate the resulting expression and get (16)

$$
V \frac{d S}{d t} \Rightarrow V=-m \cdot f \cdot g \int d t-m\left(\frac{V_{C 1}}{m}-f \cdot g\right) \int e^{-\frac{t}{m}}+C_{2},
$$

where $\mathrm{C}_{2}$ - second arbitrary constant. Accordingly, we can write the expression (15) as the equation (17) after integration

$$
S=-m \cdot f \cdot g \cdot t+m^{2}\left(\frac{V_{C 1}}{m}-f \cdot g\right) e^{-\frac{t}{m}}+C_{2} .
$$

Since the seeds are fed into the seed cut in portions at certain intervals of time $\Delta t$, then the expression of the path traveled, will look like this (18)

$$
S_{M}=(-m \cdot f \cdot g)(t-\Delta t)+m^{2} \cdot\left(\frac{V_{C 1}}{m}-f \cdot g\right) e^{-\frac{t}{m}}
$$

The interval of the traveled path, i.e. the optimal distance between the seeds in the seed line is determined by the difference of the traveled path $S_{\Delta t}$ and $S$ by the formula (19).

$$
S_{M}=(-m \cdot f \cdot g) \Delta t+m^{2} \cdot\left(\frac{V_{C 1}}{m}-f \cdot g\right)\left(1-e^{-\frac{t}{m}}\right) e^{-\frac{t}{m}}
$$

However, the straight, horizontal section of the seed duct has a very small distance, so in the future we consider the movement of seeds along the inclined plane (Fig. 6.). Taking into account the friction force on the inclined surface of the seed duct when the seeds move under the influence of gravity (Fig. 5.) the equation of seed motion will take the following form (20).

$$
m \frac{d^{2} s}{d t^{2}}=m \frac{d V}{d t}=-m \cdot g \cdot \sin \alpha-f \cdot m \cdot g \cdot \cos \alpha-V_{C}
$$

where $\mathrm{m}$ - seed mass; $\mathrm{g}$ - free falling acceleration; $\alpha$ - angle of inclination of the seed duct relatively to the horizon; $\mathrm{f}$ - coefficient of seed friction along the seed duct.

Then the time for the complete transfer of seeds to the plowshare group is determined from the expression (21).

$$
t=m \cdot \int \frac{d V_{c}}{V_{c}}+C_{1} .
$$

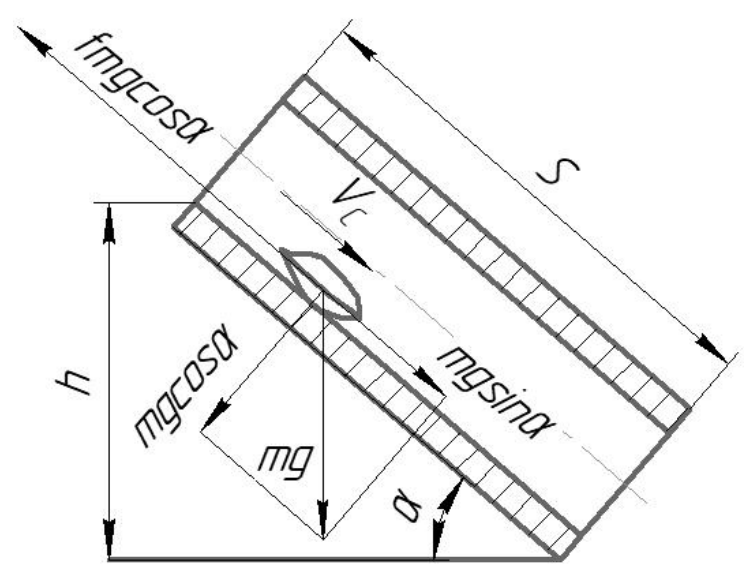

Fig. 5. Movement of seeds on the inclined plane 


\section{Conclusion}

After analyzing the movement of seeds in the seed duct, the equations of motion of the seed material in the straight and inclined sections of the seed duct are obtained. To a greater extent, the movement time is affected by the weight of the sown portion and the initial speed of the seeds when falling from the seeder. Also, in straight sections, the time depends on the geometric parameters of the seed duct.

In the future, the formula of the complete transfer of seeds from the seeder to the plowshare group will allow us to adjust the mode of adjusting the response time of the dosing system on a self-propelled seeder with a multi-level seeding system.

The study was carried out with the financial support of the Russian Foundation of Basic Researches in the framework of scientific project No. 19-316-90038.

\section{References}

1. Chikildin, V.N. Improving the process of sowing seeds of row crops with a pneumatic seeder: on the example of corn, SSAU, 2011.

2. Moskovsky M.N., Litvinov M.A., Pakhomov I.V. (2019) ITNO-2019, 43, 339-342 (2019).

3. Litvinov M.A., Adamyan A.A., Moskovsky M.N. Engineering journal of Don. 7, p 3, (2019).

4. Golubev V.V., Firsov A.S. Agricultural machinery and energy supply 3(3), 27-39 (2014).

5. Fedorenko I.Ya. Bulletin of Altai State Agrarian University 9 (167), 145-150 (2018).

6. Targ, S.M. Short course in theoretical mechanics (High School, 1986). 